\title{
Double-Expressor Lymphoma
}

National Cancer Institute

\section{Source}

National Cancer Institute. Double-Expressor Lymphoma. NCI Thesaurus. Code C138899.

A diffuse large B-cell lymphoma characterized by double expression of MYC and BCL2 proteins without MYC and BCL2 gene aberrations. These lymphomas may have a worse prognosis than other diffuse large B-cell lymphomas, not otherwise specified, but they are not as aggressive as the high-grade B-cell lymphomas, with rearrangements of MYC and BCL2 and/or BCL6 genes. 